\title{
Дисперсионный транспорт водорода в МОП-структурах после ионизирующего облучения
}

\author{
(C) O.В. Александров \\ Санкт-Петербургский государственный электротехнический университет „ЛЭТИ“, \\ 197376 Санкт-Петербург, Россия \\ E-mail: Aleksandr_ov@mail.ru
}

Поступила в Редакцию 6 апреля 2020 г.

В окончательной редакции 29 апреля 2020 г.

Принята к публикации 22 мая 2020 г.

\begin{abstract}
Показано, что описание дисперсионного транспорта ионов $\mathrm{H}^{+}$на основе модели многократного захвата позволяет количественно описать кинетику образования поверхностных состояний в МОП-структурах после ионизирующего облучения. Проведено моделирование временны́х зависимостей плотности поверхностных состояний от толщины подзатворного диэлектрика, напряженности и полярности электрического поля. Показано, что кинетика образования поверхностных состояний определяется уровнями локализованных состояний ионов водорода в диапазоне 0.76-0.98эВ, концентрацией ловушек как в объеме, так и в приграничной к подложке области $\mathrm{SiO}_{2}$ и зависит от начального распределения ионов $\mathrm{H}^{+}$.
\end{abstract}

Ключевые слова: дисперсионный транспорт, МОП-структура, ионизирующее облучение, поверхностные состояния, моделирование.

DOI: 10.21883/FTP.2020.10.49938.9403

\section{1. Введение}

При ионизирующем облучении (ИО) МОП-структур образуются положительный объемный заряд в подзатворном диэлектрике и поверхностные состояния (ПС) на межфазной границе (МФГ) $\mathrm{Si}-\mathrm{SiO}_{2}$, влияющие на электрические характеристики приборов [1-3]. Установлено, что образование объемного заряда связано с захватом дырок, образующихся при ИО, на дырочные ловушки - кислородные вакансии в структуре $\mathrm{SiO}_{2}$. Образование ПС по модели МакЛина [4] объясняется освобождением ионов водорода $\mathrm{H}^{+}$с водородосодержащих ловушек в объеме $\mathrm{SiO}_{2}$, их транспортом к МФГ $\mathrm{Si}-\mathrm{SiO}_{2}$ и депассивацией $P_{b} \mathrm{H}$-центров по реакции [5]:

$$
P_{b} \mathrm{H}+\mathrm{H}^{+} \rightarrow P_{b}+\mathrm{H}_{2} .
$$

В отличие от объемного заряда, образование ПС продолжается длительное время после окончания импульса ИО $[1,2]$. В работах [6-8] этот эффект был объяснен дисперсионной природой транспорта ионов $\mathrm{H}^{+}$в пленке аморфного диоксида кремния $\alpha-\mathrm{SiO}_{2}$. При своем перемещении в неупорядоченной структуре $\alpha-\mathrm{SiO}_{2}$ ион $\mathrm{H}^{+}$ попадает в потенциальные ямы разной глубины (локализованные состояния), в которых задерживается на время, зависящее от глубины ямы. В работах $[6,7]$ такой дисперсионный транспорт описывается моделью случайных блужданий с непрерывным временем (continuous time random walk (CTRW)), развитой Шером и Монтролом [9]. В рамках модели CTRW Саксом и Брауном [6,7] было получено описание зависимости относительной плотности ПС от времени после импульса ИО и было показано выполнение степенных зависимостей среднего времени образования ПС от толщины подзатворного оксида: $t_{1 / 2} \sim d^{n}(n=1 / \alpha, \alpha-$ параметр дисперсионности транспорта водорода, $0<\alpha<1)$ и от напряженности электрического поля: $t_{1 / 2} \sim E^{-m / \alpha}$ (m - показатель степени зависимости длины скачка от напряженности поля, $0<m<1)$. Из сравнения теории с экспериментальными зависимостями было найдено $\alpha=0.38, n=1 / \alpha=2.6$, $m=0.66$ [7]. Отметим, однако, что экспериментальный показатель степени $n$ имеет значительный разброс от 1.7 до $2.6[7,8]$. Зависимость длины скачка от напряженности поля не имеет физического подтверждения, а показатель $m=0.66$ подобран эмпирически для получения экспериментально наблюдаемой в $[4,7]$ зависимости $t_{1 / 2} \sim E^{-(1.6-1.73)}$. Также эмпирически подобран и коэффициент пропорциональности между реальным временем $\mathrm{t}$ и нормированным временем $\tau(t=500 \tau$ [7]). Таким образом, теория CTRW позволяет описать только форму временно́й зависимости относительной плотности поверхностных состояний.

Дисперсионный транспорт носителей заряда в неупорядоченных материалах может быть описан также моделью многократного захвата (multiplte trapping (MT)) носителей на локализованные состояния (ловушки) с диапазоном энергий связи [10]. В работах $[10,11]$ показана эквивалентность моделей MT и CTRW, а в работе [12] при описании дисперсионного транспорта дырок в $\mathrm{SiO}_{2}$ было продемонстрировано, что модель МТ имеет ряд преимуществ перед моделью CTRW. В частности, модель МТ позволяет лучше описать экспериментальные зависимости по накоплению заряда дырок при малых и длительных временах после ИО при разных температурах и напряженностях электрического поля [12]. Модель МТ физически более наглядна и имеет более простую численную реализацию.

Цель настоящей работы - описание дисперсионного транспорта ионов водорода в электрическом поле 
подзатворного $\mathrm{SiO}_{2}$ после ионизирующего облучения МОП-структур на основе модели многократного захвата.

\section{2. Описание модели}

В отличие от кристаллических тел в аморфных телах сохраняется только ближний порядок и отсутствует дальний порядок. В сетке аморфного диоксида кремния имеется разброс (дисперсия) в углах связи $\mathrm{Si}-\mathrm{O}-\mathrm{Si}$ от 120 до $180^{\circ}$ [13]. Соответственно имеется разброс в энергиях связи мостикового кислорода с ионами $\mathrm{H}^{+}$, перемещающимися прыжками от одного атома кислорода к другому [14]. Рассматриваем потенциальные ямы для ионов $\mathrm{H}^{+}$в $\mathrm{SiO}_{2}$ как локализованные состояния или ловушки. В соответствии с моделью многократного захвата непрерывное распределение локализованных состояний по энергиям заменяем на систему $k$ ловушек с дискретными уровнями $E_{b i}$, соответствующими энергии локализованных состояний или энергии связи иона $\mathrm{H}^{+} \mathrm{c}$ $i$-й ловушкой $S_{i}$. Захват ионов $\mathrm{H}^{+}$на ловушки происходит со скоростью $k_{1 i}$, а освобождение со скоростью $k_{2 i}$ согласно реакции

$$
S_{i}+\mathrm{H}^{+} \underset{k_{2 i}}{\stackrel{k_{1 i}}{\rightleftarrows}} S_{i} \mathrm{H}^{+} .
$$

При таком многократном захвате и освобождении с $k$ локализованных состояний в соответствии с реакцией (2) уравнения дисперсионного транспорта ионов $\mathrm{H}^{+}$c учетом их диффузии и дрейфа в электрическом поле МОП-структуры принимают вид:

$$
\begin{aligned}
\frac{\partial C_{H}^{+}}{\partial t}= & D_{H}^{+} \frac{\partial^{2} C_{H}^{+}}{\partial x^{2}}-\mu_{H}^{+} E \frac{\partial C_{H}^{+}}{\partial x} \\
& -C_{H}^{+} \sum_{i=0}^{k} k_{1 i} C_{S i}^{0}+\sum_{i=0}^{k} k_{2 i} C_{S H i}^{+},
\end{aligned}
$$

$\frac{\partial C_{S H i}^{+}}{\partial t}=-\frac{\partial C_{S i}^{0}}{d t}=k_{1 i} C_{S i}^{0} C_{H}^{+}-k_{2 i} C_{S H i}^{+}, \quad i=0,1,2 \ldots k$,

где $t-$ время; $x$ - координата, отсчитываемая от затвора $(x=0)$ до поверхности кремниевой подложки $(x=d, d-$ толщина подзатворного диэлектрика); $C_{H}^{+}-$концентрации свободных (нелокализованных) ионов $\mathrm{H}^{+} ; D_{H}^{+}$и $\mu_{H}^{+}-$их коэффициент диффузии и подвижность; $C_{S i}^{0}$ и $C_{S H i}^{+}-$концентрации $i$-х ловушек и локализованных на них ионов $\mathrm{H}^{+}$соответственно; $k-$ число локализованных состояний; $E-$ напряженность электрического поля. Рассматриваем случай малой концентрации $\left(C_{H}^{+} \ll C_{S i}^{0}\right)$ и невысокого градиента концентрации ионов $\mathrm{H}^{+}$, когда поле в диэлектрике можно считать однородным, $E=V_{G} / d$, где $V_{G}-$ напряжение на затворе.

Полагаем, что все ловушки захватывают ионы $\mathrm{H}^{+}$ с одинаковой скоростью: $k_{1 i}=k_{1}=\sigma_{H}^{+} v_{d}$, где $v_{d}-$ дрейфовая скорость, $v_{d}=\mu_{H}^{+} E, \sigma_{H}^{+}-$сечение захвата ионов $\mathrm{H}^{+}$на ловушки. Освобождение ионов $\mathrm{H}^{+}$с ловушек происходит со скоростью, зависящей от энергии связи иона с ловушкой $E_{b i}, k_{2 i}=v \exp \left(-\frac{E_{b i}}{k_{\mathrm{B}} T}\right)$, где $v-$ частотный фактор, соответствующий частоте колебаний атомов $\left(v=10^{12} \mathrm{c}^{-1}\right), k_{\mathrm{B}}-$ постоянная Больцмана, $T-$ температура $(T=300 \mathrm{~K})$. Полагаем, что распределение плотности локализованных состояний по энергии имеет экспоненциальный вид, характерный для некристаллических материалов [15]:

$$
C_{S i}^{0}\left(E_{b i}\right)=\frac{C_{S}^{0}}{E_{0}} \exp \left(-\frac{E_{b i}}{E_{0}}\right),
$$

где $C_{S}^{0}-$ полная концентрация ловушек; $E_{0}-$ характеристическая энергия, соответствующая ширине энергетического распределения ловушек, связанная с дисперсионным параметром $\alpha$ соотношением $\alpha=\frac{k T}{E_{0}}$.

Начальное распределение ионов $\mathrm{H}^{+}$после ИО определяется распределением исходных водородосодержащих дырочных ловушек в $\mathrm{SiO}_{2}$ [3], а также зависит от полярности напряжения во время облучения. При отрицательном напряжении на затворе ионы $\mathrm{H}^{+}$скапливаются у затвора $(x=0)$, полагаем по экспоненциальному закону:

$$
C_{H}^{+}(x, 0)=\frac{Q_{H 0}^{+}}{L_{H}} \exp \left(-\frac{x}{L_{H}}\right),
$$

а при положительном - у подложки $(x=d)$ :

$$
C_{H}^{+}(x, 0)=\frac{Q_{H 0}^{+}}{L_{H}} \exp \left(-\frac{d-x}{L_{H}}\right),
$$

где $Q_{H 0}^{+}-$начальное количество ионов $\mathrm{H}^{+}$, образовавшихся при ИО вследствие развала водородосодержащих дырочных ловушек, $L_{H}$ - ширина распределения водородосодержащих дырочных ловушек, $L_{H}=5-30$ нм [3].

Ловушки для ионов $\mathrm{H}^{+}$связаны с нарушениями структуры $\alpha-\mathrm{SiO}_{2}$ и располагаются как равномерно в объеме диэлектрика, так и неравномерно вблизи границы раздела с кремниевой подложкой:

$$
C_{S i}^{0}(x, 0)=C_{S i}^{0}\left(E_{b i}\right)\left[1+B \exp \left(-\frac{d-x}{L_{S}}\right)\right],
$$

где $L_{S}$ - ширина распределения ловушек (принимали $\left.L_{S}=L_{H}=10 \mathrm{Hм}\right), B-$ долевой коэффициент приграничной составляющей концентрации ловушек. В начальный момент все ловушки пустые:

$$
C_{S H i}^{0}(x, 0)=0 .
$$

Граничные условия соответствуют отражению ионов $\mathrm{H}^{+}$ от затвора и поглощению их на МФГ $\mathrm{Si}-\mathrm{SiO}_{2}$ :

$$
j_{H}^{+}(0, t)=0 ; \quad C_{H}^{+}(d, t)=0,
$$

где $j_{H}^{+}-$поток ионов $\mathrm{H}^{+}, j_{H}^{+}=-D_{H}^{+} \frac{\partial C_{H}^{+}}{\partial x}+\mu_{H}^{+} E C_{H}^{+}$. 
Плотность поверхностных состояний определяется количеством ионов $\mathrm{H}^{+}$, достигших МФГ $\mathrm{Si}-\mathrm{SiO}_{2}$ :

$$
N_{i t}=\int_{0}^{t} j_{H}^{+}(d, t) d t .
$$

Использовались следующие значения параметров: $D_{H}^{+}=1.0 \cdot \exp \left(-\frac{0.73}{k T}\right)[16] ; E_{0}=0.07 \ni \mathrm{B}\left(\alpha=\frac{k T}{E_{0}}=0.37\right)$, что соответствует литературному диапазону $\left(\alpha=0.32-0.38\right.$ для $\mathrm{H}^{+} \quad$ в $\left.\mathrm{SiO}_{2} \quad[6,7]\right)$. Полагаем, что сечение захвата ионов $\mathrm{H}^{+}$на локализованные состояния $\sigma_{H}^{+}$зависит от напряженности электрического поля таким же образом, как сечение захвата дырок $\sigma_{p}$ в $\mathrm{SiO}_{2}$ в сильных полях (при $E \gtrsim 1 \mathrm{MB} / \mathrm{cm}$ ): $\sigma_{p} \sim E^{-a}, \quad$ где $a=0.4-0.6 \quad[17,18] . \quad$ Принимаем $\sigma_{H}^{+}=\gamma E^{-0.5}$, где коэффициент $\gamma$ был определен из сопоставления с экспериментальными зависимостями: $\gamma=7.5 \cdot 10^{-10} \mathrm{~B}^{0.5} \cdot \mathrm{cm}^{1.5}$, что соответствует $\sigma_{H}^{+}=$ $=8 \cdot 10^{-13} \mathrm{~cm}^{2}$ при $E=1 \mathrm{MB} / \mathrm{cm}$. Минимальная $E_{b 0}=0.80$ эВ (при $\left.E=1 \mathrm{MB} / \mathrm{cm}\right) \quad$ и $\quad$ максимальная $E_{b k}=0.98$ эВ энергии локализованных состояний определялись из сопоставления с экспериментальными зависимостями при равномерном распределении энергий внутри диапазона: $E_{b i}=E_{b 0}+\left(E_{b k}-E_{b 0}\right) i / k$. Число локализованных состояний принималось $k \geq 5$, когда решение переставало зависеть от $k$ при $C_{S}^{0} \cdot k=\mathrm{const}$, где $C_{S}^{0}$ - полная концентрация ловушек $C_{S}^{0}=\Sigma_{i=0}^{k} C_{S i}^{0}$.

\section{3. Обсуждение результатов расчета}

Уравнения модели (2), (3), (11) решались численно с использованием неявной и явной разностных схем с начальными условиями (5)-(9) и граничными условиями (10). Решения сравнивались с экспериментальными данными $[7,8]$ по измерению плотности поверхностных состояний в МОП-транзисторах после окончания облучения электронами с энергией $10-40 \mathrm{MэВ} \mathrm{импульсами}$ длительностью 1.5-10 мкс. Варьировались толщина подзатворного диэлектрика, напряженность электрического поля, а также полярность напряжения затвора при и после ИО.

После окончания импульса ИО освобожденные ионы $\mathrm{H}^{+}$диффундируют и под действием электрического поля $\left(+V_{G}\right.$ на затворе $)$ дрейфуют от затвора к кремниевой подложке и захватываются на ловушки с разными энергиями, расположенными в объеме и приграничной области подзатворного диэлектрика. Распределения концентраций свободных и локализованных ионов $\mathrm{H}^{+}$по толщине подзатворного диэлектрика показаны на рис. 1 при различных временах после ИО при $E=1 \mathrm{MB} / \mathrm{cm}$. Как видно из рисунка, концентрация свободных ионов $\mathrm{H}^{+}$со временем уменьшается (кривые 1,2 ) вследствие из захвата на ловушки. Концентрация захваченных на все ловушки ионов $\mathrm{H}^{+}, C_{S H}^{+}(x)=\Sigma_{i=0}^{k} C_{S H i}^{+}(x)$, достигает в максимуме значения, которое мало меняется вплоть до $t \cong 10$ с (кривые 3,4$)$. Пакет захваченных ионов $\mathrm{H}^{+}$

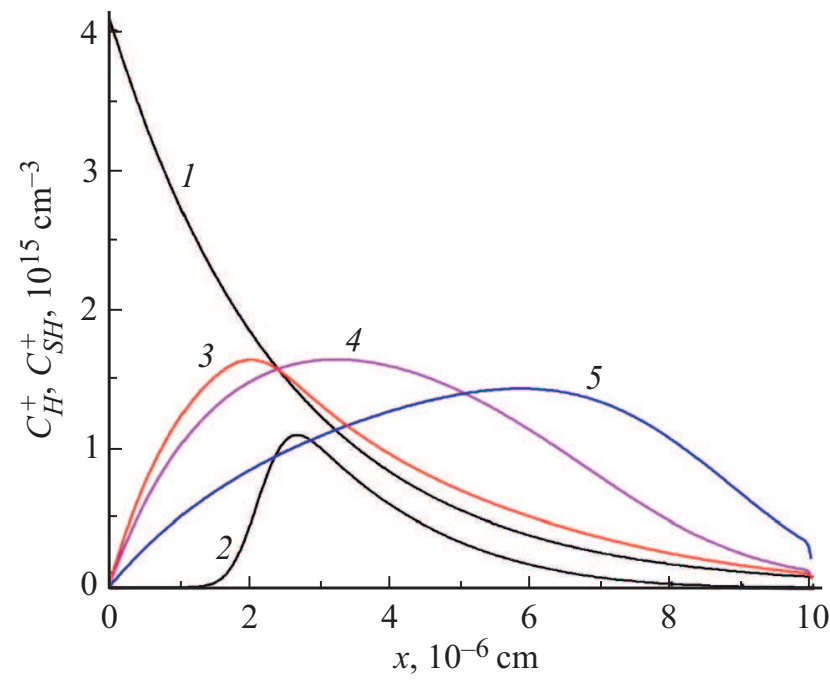

Рис. 1. Распределения по толщине $\mathrm{SiO}_{2}$ концентраций свободных $(1,2)$ и захваченных на ловушки $(3-5)$ ионов $\mathrm{H}^{+}$ при времени после ИО, с: $1-0 ; 2,3-0.1 ; 4-10$; $5-100$. Расчет при $Q_{H 0}^{+}=1 \cdot 10^{10} \mathrm{~cm}^{-2}, C_{S}^{0}=1.2 \cdot 10^{21} \mathrm{~cm}^{-3}$, $B=8, E=1 \mathrm{MB} / \mathrm{cm}$.

со временем перемещается к подложке (кривые 3-5) и при $t \cong 100$ с головная часть пакета достигает подложки. На межфазной границе с подложкой ионы $\mathrm{H}^{+}$ захватываются, их максимальная концентрация в пакете падает (кривая 5), а плотность ПС, образующихся по реакции (1), с этого времени начинает расти.

Влияние толщины подзатворного диэлектрика на кинетику образования ПС при отрицательной $(E=-1 \mathrm{MB} / \mathrm{cm})$ и положительной $(E=+1 \mathrm{MB} / \mathrm{cm})$ полярностях $V_{G}$ во время ИО и положительной полярности $V_{G}$ после ИО $(E=+1 \mathrm{MB} / \mathrm{cm})$ показано на рис. 2. По вертикали отложена относительная концентрация ПС $N_{i t} / N_{i t}^{*}$, где $N_{i t}^{*}-$ максимально возможная плотность ПС $\left(N_{i t}^{*}=Q_{H 0}^{+}\right)$. На рисунке приведены экспериментальные данные работы [8] (значки $1-5)$ и расчетные кривые $\left(1^{\prime}-5^{\prime}\right)$ с учетом дисперсии уровней ловушек от $E_{b 0}=0.80$ эВ до $E_{b k}=0.98$ эВ. Величина $E_{b 0}$ определяет начало, а $E_{b k}$ - конец роста плотности ПС во времени. Для сравнения показаны расчетные зависимости при одном уровне ловушки $E_{b 0}=E_{b k}=0.80$ эВ (кривая $\left.\sigma^{\prime}\right)$ и при отсутствии ловушек (кривая $7^{\prime}$ ). Как видно из рисунка, учет захвата водорода на ловушки приводит к существенному уменьшению скорости и увеличению времени переноса ионов $\mathrm{H}^{+}$через пленку диэлектрика. Только расчет с учетом дисперсии уровней ловушек $\left(\right.$ кривые $1^{\prime}-5^{\prime}$ ) дает удовлетворительное соответствие с экспериментом [8].

На время образования ПС оказывает влияние полярность напряжения затвора в процессе ИО. При положительной полярности $V_{G}(E=+1 \mathrm{MB} / \mathrm{cm})$ ионы $\mathrm{H}^{+}$ накапливаются у подложки и в этом случае зависимости $Q_{i t}(t)$ слабо зависят от толщины диэлектрика (кривые $\left.4^{\prime}, 5^{\prime}\right)$ по сравнению с соответствующими зависимо- 


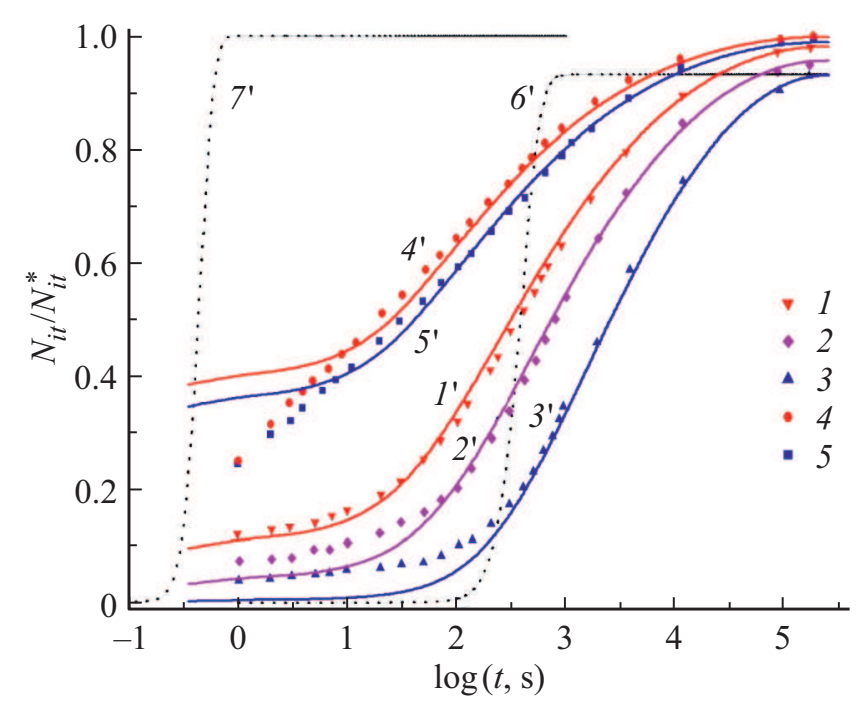

Рис. 2. Влияние на образование ПС толщины подзатворного диэлектрика, нм: $1,1^{\prime}, 4,4^{\prime}-28 ; 2,2^{\prime}-48$; $3,3^{\prime}, 5,5^{\prime}, 6^{\prime}, 7^{\prime}-104$ и полярности поля $E$ при ИО: $1,1^{\prime}-3,3^{\prime}, 6^{\prime}, 7^{\prime}-{ }^{\prime}-“, 4,4^{\prime}, 5,5^{\prime}-{ }^{\prime},+^{*} .1-5-$ эксперимент [8], сухой $\mathrm{SiO}_{2} ; 1^{\prime}-7^{\prime}-$ расчет: $1^{\prime}-6^{\prime}$ при $C_{S}^{0}=1.5 \cdot 10^{21} \mathrm{~cm}^{-3}, B=5 ; \sigma^{\prime}-$ при $E_{b 0}=E_{b k}=0.80$ эВ; $7^{\prime}-$ при $C_{S}^{0}=0$.

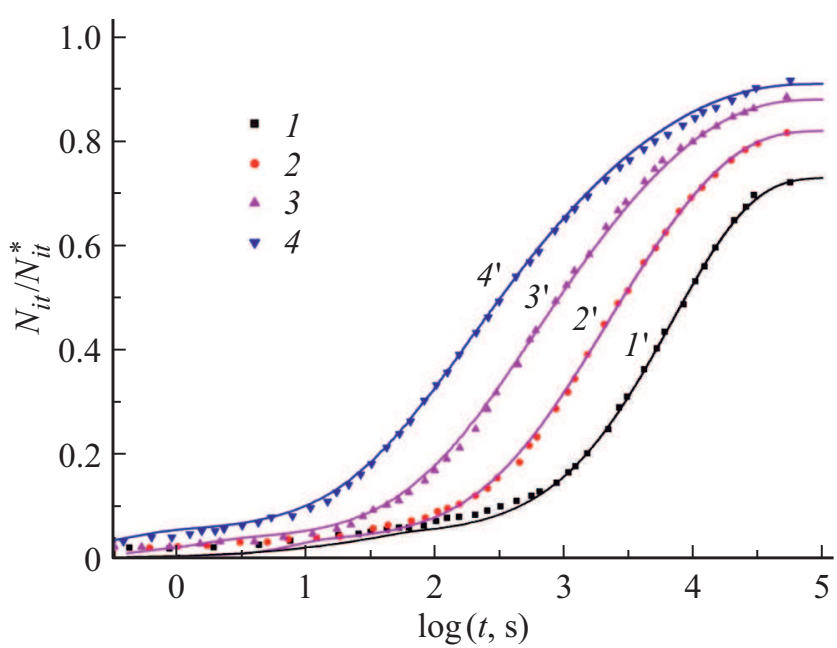

Рис. 3. Влияние на образование ПС напряженности электрического поля, МВ/см: $1,1^{\prime}-0.56 ; 2,2^{\prime}-1.07 ; 3,3^{\prime}-2.07$; $4,4^{\prime}$ - 4.08. 1-4 - эксперимент [7], влажный $\mathrm{SiO}_{2} ; 1^{\prime}-4^{\prime}-$ расчет при $E_{b 0}$, эВ: $1^{\prime}-0.86,2^{\prime}-0.83,3-0.80,4^{\prime}-0.76$. $\left(C_{S}^{0}=2.3 \cdot 10^{21} \mathrm{~cm}^{-3}, B=3.3\right)$.

стями, полученными при отрицательной полярности $V_{G}$ $(E=-1 \mathrm{MB} / \mathrm{cm})$ в процессе ИО (кривые $\left.1,1^{\prime}-3,3^{\prime}\right)$. В первом случае захват ионов $\mathrm{H}^{+}$на ловушки происходит только в приграничном к подложке слое $\mathrm{SiO}_{2}$, тогда как во втором случае (при $\left.-V_{G}\right)$ захват происходит также и по всей толщине диэлектрика.

С увеличением толщины диэлектрика среднее время образования ПС $t_{1 / 2}$ (на уровне $50 \%$ ) увеличивается. Степень зависимости $t_{1 / 2}$ от толщины зависит от концентрации ловушек как в объеме, так и в приграничном к подложке слое $\mathrm{SiO}_{2}$. Разными соотношениями между этими составляющими, зависящими от технологии получения подзатворного $\mathrm{SiO}_{2}$, можно объяснить разброс в показателях степени в зависимости $t_{1 / 2} \sim d^{n}$ от $n=1.7$ до $n=2.6$, наблюдавшийся в работах $[7,8]$. Отметим, что минимальная и максимальная энергии ловушек $E_{b i}$ $\left(E_{b 0}=0.80\right.$ эВ,$E_{b n}=0.98$ эВ $)$ примерно соответствуют экспериментально определенным эффективным энергиям активации переноса ионов $\mathrm{H}^{+}$в $\mathrm{SiO}_{2}$, лежащим, по данным работы [16], в диапазоне 0.70-0.92 эВ.

Влияние электрического поля на образование ПС после ИО показано на рис. 3 (кривые $1^{\prime}-4^{\prime}$ ) в сравнении с экспериментальными данными [7] при $d=48$ нм (значки 1-4). Как видно из рисунка, с увеличением напряженности электрического поля среднее время образования ПС сокращается. Это происходит как за счет увеличения дрейфовой скорости ионов $v_{d}=\mu_{H}^{+} E$, так и вследствие понижения эффективной высоты потенциального барьера под действием поля по корневой зависимости (эффект Пула-Френкеля [19]). Найденная зависимость имеет вид: $E_{b 0}(E)=0.92-8.6 \cdot 10^{-5} \cdot E^{0.5}$, эВ, что соответствует изменению $E_{b 0}$ от 0.86 эВ при $0.5 \mathrm{MB} / \mathrm{cm}$ до 0.75 эВ при $4 \mathrm{MB} / \mathrm{cm}$. Таким образом, зависимость среднего времени образования ПС от напряженности поля имеет более сложный вид, чем степенной.

\section{4. Заключение}

Показано, что описание дисперсионного транспорта ионов $\mathrm{H}^{+}$на основе модели многократного захвата позволяет численно описать кинетику образования поверхностных состояний в МОП-структурах после ионизирующего облучения. На основе модели МТ проведено моделирование временны́х зависимостей плотности ПС от толщины подзатворного диэлектрика, напряженности и полярности электрического поля. Показано, что кинетика образования ПС определяется уровнями локализованных состояний $\mathrm{H}^{+}$в $\mathrm{SiO}_{2}$ в диапазоне 0.76 (при $E=4 \mathrm{MB} / \mathrm{cm})$ до 0.98 эВ, что примерно соответствует экспериментально определенным эффективным энергиям активации переноса ионов $\mathrm{H}^{+}$в $\mathrm{SiO}_{2}(0.70-0.92$ эВ по данным [16]). Зависимость от толщины определяется концентрацией локализованных состояний (ловушек) как в объеме, так и в приграничной к подложке области диэлектрика. Зависимость от напряженности электрического поля связана с понижением высоты потенциального барьера и полевой зависимостью сечения захвата. Влияние полярности напряжения на затворе в процессе ИО определяется расположением начального распределения ионов $\mathrm{H}^{+}$либо у затвора, либо у подложки.

\section{Благодарности}

Автор выражает благодарность В.И. Шаповалову за полезное обсуждение.

\section{Конфликт интересов}

Авторы заявляют, что у них нет конфликта интересов. 


\section{Список литературы}

[1] T.R. Oldham, F.B. McLean. IEEE Trans. Nucl. Sci., 50 (3), 483 (2003).

[2] К.И. Таперо, В.Н. Улимов, А.М. Членов. Радиационные эфбекты в кремниевых интегральных схемах космического применения ( М., БИНОМ, 2012).

[3] О.В. Александров. ФТП, 49 (6), 793 (2015).

[4] F.B. McLean. IEEE Trans. Nucl. Sci., 27 (6), 1651 (1980).

[5] E. Cartier, J.H. Stathis, D.A. Buchanan. Appl. Phys. Lett., 63 (11), 1510 (1993).

[6] N.S. Saks, D.B. Brown. IEEE Trans. Nucl. Sci., 36 (6), 1848 (1989).

[7] D.B. Brown, N.S. Saks. J. Appl. Phys., 70 (7), 3734 (1991).

[8] M.R. Shaneyfelt, J.R. Schwank, D.M. Fleetwood, P.S. Winokur, K.L. Hughes, G.L. Hash, M.P. Connors. IEEE Trans. Nucl. Sci., 39 (6), 2244 (1992).

[9] H. Scher, E.W. Montrol. Phys. Rev. B, 12 (6), 2455 (1975).

[10] J. Noolandi. Phys. Rev. B, 16 (10), 4466 (1977).

[11] B. Hartenstein, A. Jakobs, K.W. Kehr. Phys. Rev. B, 54 (12), 8574 (1996).

[12] O.L. Curtis, J.R. Srour. J. Appl. Phys., 48 (9), 3819 (1977).

[13] Г.Я. Красников, Н.А. Зайцев. Система кремний-диоксид кремния субмикронных СБИС (М., Техносфера, 2003) c. 152.

[14] H.A. Kurtz, S.P. Karna. IEEE Trans. Nucl. Sci., 46 (6), 1574 (1999).

[15] V.I. Arkhipov, A.I. Rudenko. Phill. Mag. B, 45 (2), 189, 209 (1982).

[16] S.R. Hofstein. IEEE Trans. Electron Dev., 14 (11), 749 (1967).

[17] J.J. Tzou, J.Y.-C. Sun, C.-T. Sah. Appl. Phys. Lett., 43 (9), 861 (1983).

[18] R.J. Krantz, L.W. Aukerman, T.C. Zietlow. IEEE Trans. Nucl. Sci., 34 (6), 1196 (1987).

[19] В.А. Гуртов, П.А. Райкерус, А.А. Сарен. Зарядоперенос в структурах с диэлектрическими слоями (Петрозаводск, ПетрГУ, 2010).

Редактор Г.А. Оганесян

\section{Dispersive transport of hydrogen in MOS-structures after the ionizing radiation}

\section{O.V. Aleksandrov}

St. Petersburg State Electrotechnical University „LETl“, 197376 St. Petersburg, Russia

Abstract It is shown that the description of dispersive transport of $\mathrm{H}^{+}$ions on the base of multi-trapping model allows to quantitatively describe kinetic of the surface states formation in MOS-structures after the ionizing radiation. Modeling of time dependences of surface states density on thickness of gate dielectric, electric field strength and its polarity is carried out. It is shown that the formation kinetic of surface states is defined by the localized states levels of hydrogen ions in range from 0.76 to $0.98 \mathrm{eV}$, by a concentration of traps both in volume and in border to substrate region of $\mathrm{SiO}_{2}$, and depend on initial distribution of $\mathrm{H}^{+}$ions. 